\title{
ON EXTENDED CAPUTO FRACTIONAL DERIVATIVE OPERATOR
}

\author{
GAUHAR RAHMAN, KOTTAKKARAN SOOPPY NISAR*, MUHAMMAD ARSHAD
}

ABSTRACT. The main objective of this present paper is to introduce further extension of extended Caputo fractional derivative operator and establish the extension of an extended fractional derivative of some known elementary functions. Also, we investigate the extended fractional derivative of some familiar special functions, the Mellin transforms of newly defined Caputo fractional derivative operator and generating relations for extension of extended hypergeometric functions.

\section{INTRODUCTION AND PRELIMINARIES}

Recently, the researchers (see e. g., [2-4, 9-11, 14]) introduced various extension and generalization of various special functions. In [2] authors introduced the extended beta function and is defined by

$$
\beta\left(\tau_{1}, \tau_{2} ; p\right)=\beta_{p}\left(\tau_{1}, \tau_{2}\right)=\int_{0}^{\infty} t^{\tau_{1}-1}(1-t)^{\tau_{2}-1} e^{-\frac{p}{t(1-t)}} d t
$$

(where $\Re(p)>0, \Re\left(\tau_{1}\right)>0, \Re\left(\tau_{2}\right)>0$ ) respectively. When $p=0$, then $\beta\left(\tau_{1}, \tau_{2} ; 0\right)=$ $\beta\left(\tau_{1}, \tau_{2}\right)$.

Also, the extended hypergeometric and confluent hypergeometric functions by using the definition of extended beta function $\beta_{p}\left(\tau_{1}, \tau_{2}\right)$ is given in [3] as follows:

$$
F_{p}\left(\tau_{1}, \tau_{2} ; \tau_{3} ; z\right)=\sum_{n=0}^{\infty} \frac{\beta_{p}\left(\tau_{2}+n, \tau_{3}-\tau_{2}\right)}{\beta\left(\tau_{2}, \tau_{3}-\tau_{2}\right)}\left(\tau_{1}\right)_{n} \frac{z^{n}}{n !}
$$

where $p \geq 0$ and

$$
\Phi_{p}\left(\tau_{2} ; \tau_{3} ; z\right)=\sum_{n=0}^{\infty} \frac{\beta_{p}\left(\tau_{2}+n, \tau_{3}-\tau_{2}\right)}{\beta\left(\tau_{2}, \tau_{3}-\tau_{2}\right)} \frac{z^{n}}{n !}
$$

where $p \geq 0$.

In the same paper, they defined the following integral representations of extended hypergeometric and confluent hypergeometric functions as

$$
F_{p}\left(\tau_{1}, \tau_{2} ; \tau_{3} ; z\right)=\frac{1}{\beta\left(\tau_{2}, \tau_{3}-\tau_{2}\right)} \int_{0}^{1} t^{\tau_{2}-1}(1-t)^{\tau_{3}-\tau_{2}-1}(1-z t)^{-\tau_{1}} \exp \left(\frac{-p}{t(1-t)}\right) d t
$$

2010 Mathematics Subject Classification. 26A33,33C20, 33C15, 33C05, 33C65.

Key words and phrases. Hypergeometric function, beta function, Extended hypergeometric function, Mellin transform, fractional derivative, Caputo fractional derivative, Appell's function, generating relation.

${ }^{*}$ Corresponding author. 


$$
\left(p \geq 0, \Re\left(\tau_{3}\right)>\Re\left(\tau_{2}\right)>0,|\arg (1-z)|<\pi\right),
$$

and

$$
\begin{gathered}
\Phi_{p}\left(\tau_{2}, \tau_{3} ; \gamma ; z\right)=\frac{1}{\beta\left(\tau_{2}, \tau_{3}-\tau_{2}\right)} \int_{0}^{1} t^{\tau_{2}-1}(1-t)^{\tau_{3}-\tau_{2}-1} \exp \left(z t-\frac{-p}{t(1-t)}\right) d t, \\
\left(p \geq 0, \Re\left(\tau_{3}\right)>\Re\left(\tau_{2}\right)>0\right) .
\end{gathered}
$$

The extended Appell's function is defined by (see [10])

$$
F_{1}\left(\tau_{1}, \tau_{2}, \tau_{3} ; \tau_{4} ; x, y ; p\right)=\sum_{n=0}^{\infty} \frac{\beta_{p}\left(\tau_{1}+m+n, \tau_{4}-\tau_{1}\right)}{\beta\left(\tau_{1}, \tau_{4}-\tau_{1}\right)}\left(\tau_{2}\right)_{m}\left(\tau_{3}\right)_{n} \frac{x^{m} y^{n}}{m ! n !}
$$

where $p \geq 0$ and its integral representation by

$$
\begin{aligned}
F_{1}\left(\tau_{1}, \tau_{2}, \tau_{3} ; \tau_{4} ; x, y ; p\right) & =\frac{1}{\beta\left(\tau_{1}, \tau_{4}-\tau_{1}\right)} \int_{0}^{1} t^{\tau_{1}-1}(1-t)^{\tau_{4}-\tau_{1}-1}(1-x t)^{-\tau_{2}}(1-y t)^{-\tau_{3}} \\
& \times \exp \left(\frac{-p}{t(1-t)}\right) d t, \\
& \left(p \geq 0, \Re\left(\tau_{4}\right)>\Re\left(\tau_{1}\right)>0,|\arg (1-x)|<\pi,|\arg (1-y)|<\pi\right) .
\end{aligned}
$$

It is clear that when $p=0$, then the equations (1.2)-(1.7) reduce to the well known hypergeometric, confluent hypergeometric and Appell's series and their integral representation respectively (see [18]). Parmar et al. [15] introduced the following extended beta function as

$$
\beta_{v}\left(\tau_{1}, \tau_{2} ; p\right)=\sqrt{\frac{2 p}{\pi}} \int_{0}^{1} t^{\tau_{1}-\frac{3}{2}}(1-t)^{y-\frac{3}{2}} K_{v+\frac{1}{2}}\left(\frac{p}{t(1-t)}\right) d t
$$

where $K_{v+\frac{1}{2}}($.$) is the modified Bessel function of order v+\frac{1}{2}$. Clearly, when $v=0$ then (1.8) reduces to (1.1) by using the fact that $K_{\frac{1}{2}}(z)=\sqrt{\frac{\pi}{2 z}} e^{-z}$. In the same paper, they [15] defined the following extended hypergeometric and confluent hypergeometric functions and their integral representation respectively as:

$$
\begin{gathered}
F_{p, v}\left(\tau_{1}, \tau_{2} ; \tau_{3} ; z\right)=\sum_{n=0}^{\infty}\left(\tau_{1}\right)_{n} \frac{\beta_{v}\left(\tau_{2}+n, \tau_{3}-\tau_{2} ; p\right)}{\beta\left(\tau_{2}, \tau_{3}-\tau_{2}\right)} \frac{z^{n}}{n !} \\
\left(p, v \geq 0, \Re\left(\tau_{3}>\tau_{2}>0,|z|<1\right) .\right. \\
\Phi_{p, v}\left(\tau_{2} ; \tau_{3} ; z\right)=\sum_{n=0}^{\infty} \frac{\beta_{v}\left(\tau_{2}+n, \tau_{3}-\tau_{2} ; p\right)}{\beta\left(\tau_{2}, \tau_{3}-\tau_{2}\right)} \frac{z^{n}}{n !} \\
F_{p, v}\left(\tau_{1}, \tau_{2} ; \tau_{3} ; z\right)=\sqrt{\frac{2 p}{\pi}} \frac{1}{\beta\left(\tau_{2}, \tau_{3}-\tau_{2}\right)} \\
\times \int_{0}^{1} t^{\tau_{2}-\frac{3}{2}}(1-t)^{\tau_{3}-\tau_{2}-\frac{3}{2}}(1-z t)^{-\tau_{1}} K_{v+\frac{1}{2}}\left(\frac{p}{t(1-t)}\right) d t
\end{gathered}
$$




$$
\left(p, v \geq 0, \Re\left(\tau_{3}>\tau_{2}>0,|\arg (1-z)|<\pi\right) .\right.
$$

and

$$
\begin{aligned}
& \Phi_{p, v}\left(\tau_{2} ; \tau_{3} ; z\right)= \sqrt{\frac{2 p}{\pi}} \frac{1}{\beta\left(\tau_{2}, \tau_{3}-\tau_{2}\right)} \\
& \times \int_{0}^{1} t^{\tau_{2}-\frac{3}{2}}(1-t)^{\tau_{3}-\tau_{2}-\frac{3}{2}} \exp (z t) K_{v+\frac{1}{2}}\left(\frac{p}{t(1-t)}\right) d t \\
& \quad\left(p, v \geq 0, \Re\left(\tau_{3}>\tau_{2}>0\right) .\right.
\end{aligned}
$$

It is clear that, when $v=0$ then the equations (1.9)-(1.12) reduce to the extended hypergeometric, confluent hypergeometric functions and their integral representations defined in (1.2)-(1.5) respectively by using the fact that $K_{\frac{1}{2}}(z)=\sqrt{\frac{\pi}{2 z}} e^{-z}$.

Recently Dar and Paris [5] have introduced the following Appell's hypergeometric function by

$$
\begin{aligned}
F_{1, p, v}\left(\tau_{1}, \tau_{2}, \tau_{3} ; \tau_{4} ; x, y\right) & =F_{1, v}\left(\tau_{1}, \tau_{2}, \tau_{3} ; \tau_{4} ; x, y ; p\right) \\
= & \sum_{m, n=0}\left(\tau_{2}\right)_{m}\left(\tau_{3}\right)_{n} \frac{\beta\left(\tau_{1}+m+n, \tau_{4}-\tau_{1}\right)}{\beta\left(\tau_{1}, \tau_{4}-\tau_{1}\right)} \frac{x^{m} y^{n}}{n ! m !},
\end{aligned}
$$

where $|x|<1,|y|<1, \tau_{1}, \tau_{2}, \tau_{3}, \tau_{4} \in \mathbb{C}, \tau_{4} \neq=0,-1,-2,-3, \cdots$. In the same paper, they [5] defined its integral representation as:

$$
\begin{aligned}
F_{1, p, v}\left(\tau_{1}, \tau_{2}, \tau_{3} ; \tau_{4} ; x, y\right) & =\sqrt{\frac{2 p}{\pi}} \frac{1}{\beta\left(\tau_{1}, \tau_{4}-\tau_{1}\right)} \\
& \times \int_{0}^{1} t^{\tau_{1}-\frac{3}{2}}(1-t)^{\tau_{4}-\tau_{1}-\frac{3}{2}}(1-t x)^{-\tau_{2}}(1-t y)^{-\tau_{3}} \\
& \times K_{v+\frac{1}{2}}\left(\frac{p}{t(1-t)}\right) d t
\end{aligned}
$$

where $\Re(p) \geq 0, v \geq 0, \Re\left(\tau_{4}\right)>\Re\left(\tau_{1}\right)>0,|\arg (1-x)|<\pi$ and $|\arg (1-y)|<\pi$. Obviously, when $v=0$ in (1.13) and (1.14) then we get the extended Appell function and its integral representation (see e. g., (1.6) and (1.7)) by using the fact that $K_{\frac{1}{2}}(z)=$ $\sqrt{\frac{\pi}{2 z}} e^{-z}$.

Very recently Rahman et al. [17] introduced the extension of extended fractional derivative operator of Riemann-Liouville as:

$$
\mathfrak{D}_{x}^{\mu}\{f(x) ; p, v\}=\sqrt{\frac{2 p x^{2}}{\pi}} \frac{1}{\Gamma(-\mu)} \int_{0}^{x} f(t) t^{-\frac{1}{2}}(x-t)^{-\mu-\frac{3}{2}} K_{v+\frac{1}{2}}\left(\frac{p x^{2}}{t(x-t)}\right) d t,
$$

where $\Re(\mu)>0, \Re(p)>0$ and $v \geq 0$. It is clear that, if $v=0$, then definition 1.15 reduces to extended fractional derivative defined in [10] by using the fact that $K_{\frac{1}{2}}(z)=\sqrt{\frac{\pi}{2 z}} e^{-z}$.

\section{EXtension of HyPERGEOMETRIC FUnCtions}

In this section, we define further extension of hypergeometric and Appell's hypergeometric functions. 
Definition 2.1. The extension of extended hypergeometric function is defined as:

$$
{ }_{2} F_{1, v}(\beta, \eta ; \mu ; z ; p)=\sum_{n=0}^{\infty} \frac{(\beta)_{n}(\eta)_{n}}{(\eta-m)_{n}} \frac{\beta_{v}(\eta-m+n, m-\eta+\mu ; p)}{\beta(\eta-m, \mu-\eta+m)} \frac{z^{n}}{n !},
$$

where $m-1<\Re(\eta-\mu)<m<\Re(\eta)>0, \Re(p)>0, v \geq 0$ and $|z|<1$.

Definition 2.2. The extension of Appell's hypergeometric function is defined as:

$$
\begin{aligned}
& F_{1, v}(\eta ; \alpha, \beta ; \mu ; a z ; b z ; p) \\
= & \sum_{n, k=0}^{\infty} \frac{(\alpha)_{n}(\beta)_{n}(\eta)_{n+k}}{(\eta-m)_{n+k}} \frac{(a z)^{n}}{n !} \frac{(b z)^{k}}{k !} \frac{\beta_{v}(\eta+m+n+, \mu-\eta ; p)}{\beta(\eta-m, m+\mu-\eta)},
\end{aligned}
$$

where $m-1<\Re(\eta-\mu)<m<\Re(\eta), \Re(p)>0, v \geq 0$.

The Integral representations of (2.1) and (2.2) are defined respectively as:

$$
\begin{aligned}
{ }_{2} F_{1, v}\left(\tau_{1}, \tau_{2} ; \tau_{3} ; z ; p\right) & =\sqrt{\frac{2 p}{\pi}} \frac{1}{\beta\left(\tau_{2}-m, \tau_{3}-\tau_{2}+m\right)} \int_{0}^{1} t^{\tau_{2}-m-\frac{3}{2}}(1-t)^{\tau_{3}-\tau_{2}+m-\frac{3}{2}} \\
\times & K_{v+\frac{1}{2}}\left(\frac{p}{t(1-t)}\right){ }_{2} F_{1}\left(\tau_{1}, \tau_{2} ; \tau_{2}-m ; z t\right) d t
\end{aligned}
$$

and

$$
\begin{aligned}
F_{1, v}\left(\tau_{1}, \tau_{2}, \tau_{3} ; \tau_{4} ; x, y ; p\right) & =\sqrt{\frac{2 p}{\pi}} \frac{1}{\beta\left(\tau_{1}-m, \tau_{4}-\tau_{1}+m\right)} \int_{0}^{1} t^{\tau_{1}-m-\frac{3}{2}}(1-t)^{\tau_{4}-\tau_{1}-m-\frac{3}{2}} \\
\times & K_{v+\frac{1}{2}}\left(\frac{p}{t(1-t)}\right) F_{1}\left(\tau_{1}, \tau_{2} ; \tau_{3} ; \tau_{1}-m ; x t ; y t\right) d t
\end{aligned}
$$

Remark 2.1. If we letting $v=0$, then the equations (2.1)-(2.4) respectively reduce to the extended hypergeometric functions ${ }_{2} F_{1}$ and $F_{1}$ and their integral representations (see [6]).

\section{EXTENSION OF FRACTIONAL DERIVATIVE OPERATOR}

Recently, the application and importance of fractional calculus have been paid more attentions. In the field of mathematical analysis, the fractional calculus is a more helpful tool to find out differentials and integrals with the real numbers or with the complex numbers powers of the fractional calculus. Various extensions and generalization of fractional derivative operators are recently investigated by the researchers (see $[1,8,12,16,20])$. In this section, we define further extension of extended Caputo fractional derivative operator.

We recall the classical Caputo fractional derivative operator which is defined by

Definition 3.1. (see [7])

$$
\mathfrak{D}_{z}^{\mu}\{f(z)\}=\frac{1}{\Gamma(m-\mu)} \int_{0}^{z}(z-t)^{m-\mu-1} \frac{d^{m}}{d t^{m}} f(t) d t
$$

where $m-1<\Re(\mu)<m$ where $m=1,2, \cdots$.

Recently Kiymaz et al. [6] introduced the extended Caputo fractional derivative operator as: 


\section{Definition 3.2.}

$$
\mathfrak{D}_{z}^{\mu}\{f(z)\}=\frac{1}{\Gamma(m-\mu)} \int_{0}^{z}(z-t)^{m-\mu-1} \exp \left(-\frac{p}{t(1-t)}\right) \frac{d^{m}}{d t^{m}} f(t) d t
$$

where $m-1<\Re(\mu)<m$ where $m=1,2, \cdots$ and $\Re(p)>0$.

In view of [17], we introduce further extension of extended Caputo fractional derivative operator.

Definition 3.3. The extension of extended Caputo fractional derivative operator is defined as;

$$
\begin{aligned}
\mathfrak{D}_{z}^{\mu}\{f(z) ; p, v\} & =\mathfrak{D}_{z ; v}^{\mu ; p}\{f(z)\} \\
& =\sqrt{\frac{2 p z^{2}}{\pi}} \frac{1}{\Gamma(m-\mu)} \int_{0}^{z} t^{-\frac{1}{2}}(z-t)^{m-\mu-\frac{3}{2}} K_{v+\frac{1}{2}}\left(\frac{p z^{2}}{t(z-t)}\right) \frac{d^{m}}{d t^{m}} f(t) d t
\end{aligned}
$$

For the case $m-1<\Re(\mu)<m$ where $m=1,2, \cdots, \Re(p)>0 v \geq 0$.

Remark 3.1. Obviously if $v=0$, then definition 3.3 reduces to extended Caputo fractional derivative defined in 3.2 (see [6]) by using the fact that $K_{\frac{1}{2}}(z)=\sqrt{\frac{\pi}{2 z}} e^{-z}$.

Now, we prove some theorems involving the modified extension of fractional derivative operator.

Theorem 3.1. The following formula hold true,

$$
\mathfrak{D}_{z}^{\mu}\left\{z^{\eta} ; p, v\right\}=\frac{\Gamma(\eta+1) \beta_{v}(\eta-m+1, m-\mu ; p)}{\Gamma(\eta-\mu+1) \beta(\eta-m+1, m-\mu)} z^{\eta-\mu}, \Re(\mu)>0,
$$

where $m-1<\Re(\mu)<m$ and $\Re(\mu)<\Re(\eta)$.

Proof. From (3.3), we have

$$
\begin{aligned}
\mathfrak{D}_{z}^{\mu}\left\{z^{\eta} ; p, v\right\} & =\frac{\sqrt{\frac{2 p z^{2}}{\pi}}}{\Gamma(m-\mu)} \int_{0}^{z} t^{-\frac{1}{2}}(z-t)^{m-\mu-\frac{3}{2}} K_{v+\frac{1}{2}}\left(\frac{p z^{2}}{t(z-t)}\right) \frac{d^{m}}{d t^{m}} t^{\eta} d t \\
& =\frac{\sqrt{\frac{2 p z^{2}}{\pi}}}{\Gamma(m-\mu)}[\eta(\eta-1) \cdots(\eta-m+1)] \\
& \times \int_{0}^{z}(z-t)^{m-\mu-\frac{3}{2}} K_{v+\frac{1}{2}}\left(\frac{p z^{2}}{t(z-t)}\right) t^{\eta-m t-\frac{1}{2}} d t \\
& =\frac{\sqrt{\frac{2 p z^{2}}{\pi}} \Gamma(\eta+1)}{\Gamma(\eta-m+1) \Gamma(m-\mu)} \int_{0}^{z}(z-t)^{m-\mu-\frac{3}{2}} K_{v+\frac{1}{2}}\left(\frac{p z^{2}}{t(z-t)}\right) t^{\eta-m-\frac{1}{2}} d t .
\end{aligned}
$$

Substituting $t=u z$ in (3.5), we have

$$
\mathfrak{D}_{z}^{\mu}\left\{z^{\eta} ; p, v\right\}=\frac{\sqrt{\frac{2 p}{\pi}} \Gamma(\eta+1)}{\Gamma(\eta-m+1) \Gamma(m-\mu)} z^{\eta-\mu} \int_{0}^{z} u^{\eta-m-\frac{1}{2}}(1-u)^{m-\mu-\frac{3}{2}} K_{v+\frac{1}{2}}\left(\frac{p}{u(1-u)}\right) d u
$$


by using the definition (1.8) to the above equation, we get

$$
\begin{aligned}
\mathfrak{D}_{z}^{\mu}\left\{z^{\eta} ; p, v\right\} & =\frac{\Gamma(\eta+1)}{\Gamma(\eta-m+1) \Gamma(m-\mu)} z^{\eta-\mu} \beta_{v}(\eta-m+1, m-\mu ; p) \\
& =\frac{\Gamma(\eta+1) \beta_{v}(\eta-m+1, m-\mu ; p)}{\Gamma(\eta-\mu+1) \beta(\eta-m+1, m-\mu)} z^{\eta-\mu}
\end{aligned}
$$

which is the required result.

Theorem 3.2. Let $m-1<\Re(\mu)<m$ and suppose that the function $f(z)$ is analytic on the disk $|z|<r$ for some $r \in \mathbb{R}^{+}$and with its power series expansion given by $f(z)=$ $\sum_{n=0}^{\infty} a_{n} z^{n}$. Then

$$
\mathfrak{D}_{z}^{\mu}\{f(z) ; p, v\}=\sum_{n=0}^{\infty} a_{n} \mathfrak{D}_{z}^{\mu}\left\{z^{n} ; p, v\right\} .
$$

Proof. Using the series expansion of the function $f(z)$ in (3.3) gives

$$
\mathfrak{D}_{z}^{\mu}\{f(z) ; p, v\}=\frac{\sqrt{\frac{2 p z^{2}}{\pi}}}{\Gamma(m-\mu)} \int_{0}^{z} t^{-\frac{1}{2}}(z-t)^{m-\mu-\frac{3}{2}} K_{v+\frac{1}{2}}\left(\frac{p z^{2}}{t(z-t)}\right) \sum_{n=0}^{\infty} a_{n} \frac{d^{m}}{d t^{m}} t^{n} d t .
$$

As the series is uniformly convergent and the integrand is absolutely convergent, therefore interchanging the order of summation and integration gives

$$
\begin{aligned}
\mathfrak{D}_{z}^{\mu}\{f(z) ; p, v\} & =\sum_{n=0}^{\infty} a_{n}\left\{\frac{\sqrt{\frac{2 p z^{2}}{\pi}}}{\Gamma(m-\mu)} \int_{0}^{z} t^{-\frac{1}{2}}(z-t)^{m-\mu-\frac{3}{2}} K_{v+\frac{1}{2}}\left(\frac{p z^{2}}{t(z-t)}\right) \frac{d^{m}}{d t^{m}} t^{n} d t\right\} \\
& =\sum_{n=0}^{\infty} a_{n} \mathfrak{D}_{z}^{\mu}\left\{z^{n} ; p, v\right\},
\end{aligned}
$$

which is the required proof.

Theorem 3.3. Let $m-1<\Re(\mu)<m$ and suppose that the function $f(z)$ is analytic on the disk $|z|<r$ for some $r \in \mathbb{R}^{+}$and with its power series expansion given by $f(z)=$ $\sum_{n=0}^{\infty} a_{n} z^{n}$. Then

$$
\mathfrak{D}_{z}^{\mu}\left\{z^{\eta-1} f(z) ; p, v\right\}=\frac{\Gamma(\eta) z^{\eta-\mu-1}}{\Gamma(\eta-\mu)} \sum_{n=0}^{\infty} a_{n} \frac{(\eta)_{n}}{(\eta-m)_{n}} \frac{\beta_{v}(\eta-m+n, m-\mu ; p)}{\beta(\eta-m, m-u)} z^{n}
$$

Proof. By applying Theorem (3.2) and (3.1), we have

$$
\begin{aligned}
\mathfrak{D}_{z}^{\mu}\left\{z^{\eta-1} f(z) ; p, v\right\} & =\sum_{n=0}^{\infty} a_{n} \mathfrak{D}_{z}^{\mu}\left\{z^{\eta+n-1} ; p, v\right\} \\
& =\frac{\Gamma(\eta) z^{\eta-\mu-1}}{\Gamma(\eta-\mu)} \sum_{n=0}^{\infty} a_{n} \frac{(\eta)_{n}}{(\eta-\mu)_{n}} \frac{\beta_{v}(\eta-m+n, m-\mu ; p)}{\beta(\eta-m+n, m-u)} z^{n} \\
& =\frac{\Gamma(\eta) z^{\eta-\mu-1}}{\Gamma(\eta-\mu)} \sum_{n=0}^{\infty} a_{n} \frac{(\eta)_{n}}{(\eta-m)_{n}} \frac{\beta_{v}(\eta-m+n, m-\mu ; p)}{\beta(\eta-m, m-u)} z^{n}
\end{aligned}
$$

which is the desired result. 
Theorem 3.4. The following result holds true:

$$
\begin{aligned}
\mathfrak{D}_{z}^{\eta-\mu}\left\{z^{\eta-1}(1-z)^{-\beta} ; p, v\right\} & =\frac{\Gamma(\eta)}{\Gamma(\mu)} z^{\mu-1} \\
& \times \sum_{n=0}^{\infty} \frac{(\beta)_{n}(\eta)_{n}}{(\eta-m)_{n}} \frac{\beta_{v}(\eta-m+n, m-\eta+\mu ; p)}{\beta(\eta-m, \mu-\eta+m)} \frac{z^{n}}{n !}, \\
& =\frac{\Gamma(\eta)}{\Gamma(\mu)} z^{\mu-1}{ }_{2} F_{1, v}(\beta, \eta ; \mu ; z ; p) .
\end{aligned}
$$

where $m-1<\Re(\eta-\mu)<m<\Re(\eta)>0, \Re(p)>0, v \geq 0$.

Proof. Using the power series of $(1-z)^{-\beta}$ and applying Theorem 3.1, we have

$$
\begin{aligned}
\mathfrak{D}_{z}^{\eta-\mu}\left\{z^{\eta-1}(1-z)^{-\beta} ; p, v\right\} & =\mathfrak{D}_{z}^{\eta-\mu}\left\{z^{\eta-1} \sum_{n=0}^{\infty}(\beta)_{n} \frac{z^{n}}{n !}\right\} \\
& =\sum_{n=0}^{\infty} \frac{(\beta)_{n}}{n !} \mathfrak{D}_{z}^{\eta-\mu}\left\{z^{\eta+n-1} ; p, v\right\} \\
& =\sum_{n=0}^{\infty} \frac{(\beta)_{n}}{n !} \frac{\Gamma(\eta+n)}{\Gamma(\eta+n-m) \Gamma(m-\eta+\mu)} \\
& \times \beta_{v}(\eta-m+n, m-\eta+\mu ; p) z^{\mu+n-1} \\
& =\sum_{n=0}^{\infty} \frac{(\beta)_{n}}{n !} \frac{\Gamma(\eta+n)}{\Gamma(\mu+n)} \frac{\beta_{v}(\eta-m+n, m-\eta+\mu ; p)}{\beta(\eta-m+n, m-\eta+\mu)} z^{\mu+n-1} \\
& =\frac{\Gamma(\eta)}{\Gamma(\mu)} z^{\mu-1} \sum_{n=0}^{\infty} \frac{(\beta)_{n}(\eta)_{n}}{(\mu)_{n}} \frac{\beta_{v}\left(\eta-m+n+\frac{1}{2}, m-\eta+\mu ; p\right)}{\beta(\eta-m+n, m-\eta+\mu)} \frac{z^{n}}{n !} \\
& =\frac{\Gamma(\eta)}{\Gamma(\mu)} z^{\mu-1} \sum_{n=0}^{\infty} \frac{(\beta)_{n}(\eta)_{n}}{(\eta-m)_{n}} \frac{\beta_{v}(\eta-m+n, m-\eta+\mu ; p)}{\beta(\eta-m, \mu-\eta+m)} \frac{z^{n}}{n !},
\end{aligned}
$$

with the aid of (2.1), we get the required result.

Theorem 3.5. The following result holds true:

$$
\begin{aligned}
& \mathfrak{D}_{z}^{\eta-\mu}\left\{z^{\eta-1}(1-a z)^{-\alpha}(1-b z)^{-\beta} ; p, v\right\} \\
= & \frac{\Gamma(\eta)}{\Gamma(\mu)} z^{\mu-1} \sum_{n, k=0}^{\infty} \frac{(\alpha)_{n}(\beta)_{n}(\eta)_{n+k}}{(\eta-m)_{n+k}} \frac{(a z)^{n}}{n !} \frac{(b z)^{k}}{k !} \frac{\beta_{v}(\eta+m+n+, \mu-\eta ; p)}{\beta(\eta-m, m+\mu-\eta)}, \\
= & \frac{\Gamma(\eta)}{\Gamma(\mu)} z^{\mu-1} F_{1, v}(\eta ; \alpha, \beta ; \mu ; a z ; b z ; p),
\end{aligned}
$$

where $m-1<\Re(\eta-\mu)<m<\Re(\eta), \Re(p)>0, v \geq 0$.

Proof. To prove(3.9), we use the following power series expansion

$$
(1-a z)^{-\alpha}(1-b z)^{-\beta}=\sum_{n=0}^{\infty} \sum_{k=0}^{\infty}(\alpha)_{n}(\beta)_{k} \frac{(a z)^{n}}{n !} \frac{(b z)^{k}}{k !} .
$$


Now, applying Theorem 3.4, we obtain

$$
\begin{aligned}
& \mathfrak{D}_{z}^{\eta-\mu}\left\{z^{\eta-1}(1-a z)^{-\alpha}(1-b z)^{-\beta} ; p, \alpha\right\} \\
& =\sum_{n=0}^{\infty} \sum_{k=0}^{\infty}(\alpha)_{n}(\beta)_{k} \frac{(a)^{n}}{n !} \frac{(b)^{k}}{k !} \mathfrak{D}_{z}^{\eta-\mu}\left\{z^{\eta+n+k-1} ; p, v\right\} .
\end{aligned}
$$

Using Theorem 3.1, we have

$$
\begin{aligned}
& \mathfrak{D}_{z}^{\eta-\mu}\left\{z^{\eta-1}(1-a z)^{-\alpha}(1-b z)^{-\beta} ; p, v\right\} \\
& =\sum_{n=0}^{\infty} \sum_{k=0}^{\infty}(\alpha)_{n}(\beta)_{k} \frac{(a)^{n}}{n !} \frac{(b)^{k}}{k !} \frac{\Gamma(\eta+n+k) \beta_{v}^{\alpha}(\eta+m+n, \mu-\eta ; p)}{\Gamma(m+\mu-\eta) \Gamma(\eta-m+n+k)} z^{\mu+n+k-1} \\
& =\frac{\Gamma(\eta)}{\Gamma(\mu)} z^{\mu-1} \sum_{n=0}^{\infty} \sum_{k=0}^{\infty} \frac{(\alpha)_{n}(\beta)_{k}(\eta)_{n+k}}{(\eta-m)_{n+k}} \frac{(a z)^{n}}{n !} \frac{(b z)^{k}}{k !} \frac{\beta_{v}(\eta+m+n, \mu-\eta ; p)}{\beta(\eta-m, m+\mu-\eta)}
\end{aligned}
$$

with the aid of (2.2), we get the required result.

\section{Further Results of extended Caputo fractional Derivative operator}

In this section, we apply the extension of Caputo fractional derivative operator (3.3) to some known functions. Also, we investigate the Mellin transforms of the extension of Caputo fractional derivative operator.

Theorem 4.1. The following result holds true:

$$
\mathfrak{D}_{z}^{\mu}\left\{e^{z} ; p, v\right\}=\frac{z^{m-\mu}}{\Gamma(m-\mu)} \sum_{n=0}^{\infty} \frac{z^{n}}{n !} \beta_{v}(n+1, m-\mu ; p)
$$

for all $z$.

Proof. Using the power series of $e^{z}$ and applying Theorems 3.2 and 3.1, we have

$$
\begin{aligned}
\mathfrak{D}_{z}^{\mu}\left\{e^{z} ; p, v\right\} & =\sum_{n=0}^{\infty} \frac{z^{n}}{n !} \mathfrak{D}_{z}^{\mu}\left\{z^{n} ; p, v\right\} \\
= & \sum_{n=m}^{\infty} \frac{\Gamma(n+1) \beta_{v}(n-m+1, m-\mu ; p)}{\Gamma(n-\mu+1) \beta(n-m+1, m-\mu)} \frac{z^{n-\mu}}{n !} \\
= & \sum_{n=0}^{\infty} \frac{\Gamma(n+m+1) \beta_{v}(n+1, m-\mu ; p)}{\Gamma(n+m-\mu+1) \beta(n+1, m-\mu)} \frac{z^{n+m-\mu}}{(n+m) !} \\
= & \frac{z^{m-\mu}}{\Gamma(m-\mu)} \sum_{n=0}^{\infty} \frac{z^{n}}{n !} \beta_{v}(n+1, m-\mu ; p) .
\end{aligned}
$$

Theorem 4.2. The following result holds true:

$$
\begin{aligned}
& \mathfrak{D}_{z}^{\mu}\left\{{ }_{2} F_{1}\left(\tau_{1}, \tau_{2} ; \tau_{3} ; z\right) ; p, v\right\} \\
= & \frac{\left(\tau_{1}\right)_{m}\left(\tau_{2}\right)_{m}}{\left(\tau_{3}\right)_{m}} \frac{z^{m-\mu}}{\Gamma(1-\mu+m)} \sum_{n=0}^{\infty} \frac{\left(\tau_{1}+m\right)_{n}\left(\tau_{2}+m\right)_{n}}{\left(\tau_{3}+m\right)_{n}(1-\mu+m)_{n}} \frac{\beta_{v}(n+1, m \mu ; p) z^{n}}{\beta(n+1, m-\mu)}
\end{aligned}
$$


for all $|z|<1$.

Proof. Using the power series of Gauss hypergeometric function ${ }_{2} F_{1}($.$) and applying The-$ orems 3.2 and 3.1 , we have

$$
\begin{aligned}
& \mathfrak{D}_{z}^{\mu}\left\{{ }_{2} F_{1}\left(\tau_{1}, \tau_{2} ; \tau_{3} ; z\right) ; p, v\right\} \\
= & \sum_{n=0}^{\infty} \frac{\left(\tau_{1}\right)_{n}\left(\tau_{2}\right)_{n}}{\left(\tau_{3}\right)_{n} n !} \mathfrak{D}_{z}^{\mu}\left\{z^{n} ; p, v\right\} \\
= & \sum_{n=m}^{\infty} \frac{\left(\tau_{1}\right)_{n}\left(\tau_{2}\right)_{n}}{\left(\tau_{3}\right)_{n} n !} \frac{\Gamma(n+1) \beta_{v}(n-m+1, m-\mu ; p)}{\Gamma(n-\mu+1) \beta(n-m+1, m-\mu)} \frac{z^{n-\mu}}{n !} \\
= & \sum_{n=0}^{\infty} \frac{\left(\tau_{1}\right)_{n+m}\left(\tau_{2}\right)_{n+m}}{\left(\tau_{3}\right)_{n+m}(n+m) ! \Gamma(n+m+1) \beta_{v}(n+1, m-\mu ; p)} \frac{z^{n+m-\mu}}{(n+m) !} \\
= & \frac{\left(\tau_{1}\right)_{m}\left(\tau_{2}\right)_{m}}{\left(\tau_{3}\right)_{m}} \frac{z^{m-\mu}}{\Gamma(1-\mu+1) \beta(n+1, m-\mu)} \sum_{n=0}^{\infty} \frac{\left(\tau_{1}+m\right)_{n}\left(\tau_{2}+m\right)_{n}}{\left(\tau_{3}+m\right)_{n}(1-\mu+m)_{n}} \frac{\beta_{v}(n+1, m \mu ; p) z^{n}}{\beta(n+1, m-\mu)} .
\end{aligned}
$$

Theorem 4.3. The following result holds true:

$$
\mathfrak{D}_{z}^{\mu}\left[E_{\gamma, \delta}^{\tau}(z) ; p, v\right]=\frac{z^{m-\mu}(\tau)_{m}}{\Gamma(m-\mu)} \sum_{n=0}^{\infty} \frac{(\mu)_{n}}{\Gamma(\gamma n+\gamma m+\delta)} \beta_{v}(n+1, \mu-\eta ; p) \frac{z^{n}}{n !},
$$

where $\gamma, \delta, \tau, \in \mathbb{C}, \Re(p)>0$, and $E_{\gamma, \delta}^{\tau}(z)$ is Mittag-Leffler function (see [13]) defined as:

$$
E_{\gamma, \delta}^{\tau}(z)=\sum_{n=0}^{\infty} \frac{(\tau)_{n}}{\Gamma(\gamma n+\delta)} \frac{z^{n}}{n !} .
$$

Proof. Using (4.4) in (4.3), we have

$$
\mathfrak{D}_{z}^{\mu}\left[E_{\gamma, \delta}^{\tau}(z) ; p, v\right]=\mathfrak{D}_{z}^{\mu}\left\{\sum_{n=0}^{\infty} \frac{(\mu)_{n}}{\Gamma(\gamma n+\delta)} \frac{z^{n}}{n !} ; p, v .\right.
$$

By Theorem 3.2, we have

$$
\mathfrak{D}_{z}^{\mu}\left\{E_{\gamma, \delta}^{\tau}(z) ; p, v\right\}=\sum_{n=0}^{\infty} \frac{(\tau)_{n}}{\Gamma(\gamma n+\delta) n !}\left\{\mathfrak{D}_{z}^{\mu}\left\{z^{n} ; p, v\right\}\right\} .
$$

Applying Theorem 3.1, we get

$$
\begin{aligned}
\mathfrak{D}_{z}^{\mu}\left\{E_{\gamma, \delta}^{\mu}(z) ; p, v\right\} & =\sum_{n=m}^{\infty} \frac{(\tau)_{n}}{\Gamma(\gamma n+\delta)} \frac{\Gamma(n+1) \beta_{v}(n-m+1, m-\mu ; p)}{\Gamma(n-\mu+1) \beta(n-m+1, m-\mu)} \frac{z^{n-\mu}}{n !} \\
& =\sum_{n=0}^{\infty} \frac{(\tau)_{n+m}}{\Gamma(\gamma n+\gamma m+\delta)} \frac{\Gamma(n+m+1) \beta_{v}(n+1, m-\mu ; p)}{\Gamma(n+m-\mu+1) \beta(n+1, m-\mu)} \frac{z^{n+m-\mu}}{(n+m) !} \\
& =\frac{z^{m-\mu}(\tau)_{m}}{\Gamma(m-\mu)} \sum_{n=0}^{\infty} \frac{(\tau+m)_{n}}{\Gamma(\gamma n+\gamma m+\delta)} \frac{z^{n}}{n !} \beta_{v}(n+1, m-\mu ; p) .
\end{aligned}
$$


Theorem 4.4. The following result holds true:

$$
\begin{aligned}
& \mathfrak{D}_{z}^{\mu}\left\{{ }_{m} \Psi_{n}\left[\begin{array}{c}
\left.\left.\left(\alpha_{i}, A_{i}\right)_{1, m} ; \mid z\right] ; p, v\right\} \\
\left(\beta_{j}, B_{j}\right)_{1, n} ;
\end{array}\right]=\frac{z^{m-\mu}}{\Gamma(m-\mu)}\right. \\
& \times \sum_{k=0}^{\infty} \frac{\prod_{i=1}^{m} \Gamma\left(\alpha_{i}+A_{i} k\right)}{\prod_{j=1}^{n} \Gamma\left(\beta_{j}+B_{j} k\right)} \beta_{v}(n+1, m-\mu ; p) \frac{z^{n}}{n !},
\end{aligned}
$$

where $\Re(p)>0, v \geq 0$ and ${ }_{m} \Psi_{n}(z)$ denotes Fox-Wright function defined by (see [7], pp. 56-58)

$$
{ }_{m} \Psi_{n}(z)={ }_{m} \Psi_{n}\left[\begin{array}{c}
\left(\alpha_{i}, A_{i}\right)_{1, m} ; \\
\left(\beta_{j}, B_{j}\right)_{1, n} ;
\end{array}\right]=\sum_{k=0}^{\infty} \frac{\prod_{i=1}^{m} \Gamma\left(\alpha_{i}+A_{i} k\right)}{\prod_{j=1}^{n} \Gamma\left(\beta_{j}+B_{j} k\right.} \frac{z^{k}}{k !} .
$$

Proof. Applying Theorem 3.1 and followed the same procedure used in Theorem 4.3, we get the desired result.

Theorem 4.5. The following Mellin transform formula holds true:

$$
M\left\{\mathfrak{D}_{z ; p}^{\mu ; v}\left(z^{\eta}\right) ; p \rightarrow r,\right\}=\frac{2^{1-r} \Gamma(\eta+1) \Gamma\left(\frac{r-v}{2}\right) \Gamma\left(\frac{r+v+1}{2}\right)}{\sqrt{\pi} \Gamma(m-\mu) \Gamma(\eta-m+1)} \beta(\eta-m+r+1, m-\mu+r) z^{\eta-\mu},
$$

where $\Re(\eta)>m-1, \Re(r)>0$.

Proof. Applying the Mellin transform on definition (3.3) and Theorem 3.1, we have

$$
\begin{aligned}
M\left\{\mathfrak{D}_{z ; p}^{\mu ; v}\left(z^{\eta}\right) ; p \rightarrow r\right\} & =\int_{0}^{\infty} p^{r-1} \mathfrak{D}_{z ; p}^{\mu ; v}\left(z^{\eta}\right) d p \\
& =\int_{0}^{\infty} p^{r-1} \frac{\Gamma(\eta+1) \beta_{v}(\eta-m+1, m-\mu ; p)}{\Gamma(\eta-\mu+1)} z^{\eta-\mu} d p \\
& =\frac{\Gamma(\eta+1)}{\Gamma(\eta-m+1) \Gamma(m-\mu)} z^{\eta-\mu} \int_{0}^{\infty} p^{r-1} \beta_{v}(\eta-m+1, m-\mu ; p) d p
\end{aligned}
$$

By using the following Mellin transform of $\beta_{v}(x, y ; p)$ in 4.8 (see [15]), we get the required result.

$\int_{0}^{\infty} p^{r-1} \beta_{v}(\eta-m+1, m-\mu ; p) d p=\frac{2^{r-1}}{\sqrt{\pi}} \Gamma\left(\frac{r-v}{2}\right) \Gamma\left(\frac{r+v+1}{2}\right) \beta(\eta-m+1+r, m-\mu+r)$.

Theorem 4.6. The following Mellin transform formula holds true:

$$
\begin{aligned}
& M\left\{\mathfrak{D}_{z ; p}^{\mu ; v}\left((1-z)^{-\alpha}\right) ; p \rightarrow r\right\} \\
= & \frac{2^{1-r} \Gamma\left(\frac{r-v}{2}\right) \Gamma\left(\frac{r+v+1}{2}\right)(\alpha)_{m}}{\sqrt{\pi} \Gamma(m-\mu)} z^{m-\mu} \sum_{n=0}^{\infty}(\alpha+m)_{n} \frac{z^{n}}{n !} \beta(n+r+1, m-\mu+r),
\end{aligned}
$$

where $\Re(p)>0, \Re(r)>0$, and $|z|<1$. 
Proof. Applying Theorem 4.5 with $\eta=n$ and using the power series extension of $(1-z)^{-\alpha}$, we can write

$$
\begin{aligned}
& M\left\{\mathfrak{D}_{z ; p}^{\mu ; v}\left((1-z)^{-\alpha}\right) ; p \rightarrow r\right\}=\sum_{n=0}^{\infty} \frac{(\alpha)_{n}}{n !} M\left\{\mathfrak{D}_{z ; p}^{\mu ; \alpha}\left(z^{n}\right) ; p \rightarrow r\right\} \\
= & \frac{2^{1-r} \Gamma\left(\frac{r-v}{2}\right) \Gamma\left(\frac{r+v+1}{2}\right)}{\sqrt{\pi} \Gamma(m-\mu)} \sum_{n=m}^{\infty} \frac{(\alpha)_{n} \Gamma(n+1)}{n ! \Gamma(n-m+1)} \beta(n-m+r+1, m-\mu+r) z^{n-\mu} \\
= & \frac{2^{1-r} \Gamma\left(\frac{r-v}{2}\right) \Gamma\left(\frac{r+v+1}{2}\right)}{\sqrt{\pi} \Gamma(m-\mu)} z^{m-\mu} \sum_{n=0}^{\infty}(\alpha)_{n+m} \frac{z^{n}}{n !} \beta(n+r+1, m-\mu+r) \\
= & \frac{2^{1-r} \Gamma\left(\frac{r-v}{2}\right) \Gamma\left(\frac{r+v+1}{2}\right)(\alpha)_{m}}{\sqrt{\pi} \Gamma(m-\mu)} z^{m-\mu} \sum_{n=0}^{\infty}(\alpha+m)_{n} \frac{z^{n}}{n !} \beta(n+r+1, m-\mu+r)
\end{aligned}
$$

which is the required proof.

\section{Generating Relations}

In this section, we applying Theorems 3.4 and 3.5 and obtain generating relations for the extension of extended hypergeometric functions ${ }_{2} F_{1, v}$ and $F_{1, v}$.

Theorem 5.1. Assume that $m-1<\Re(\eta-\mu)<m<\Re(\eta)$ and $|z|<\min \{1,|1-t|\}$, then

$$
\sum_{n=0}^{\infty} \frac{(\lambda)_{n}}{n !}{ }_{2} F_{1, v}(\lambda+n, \eta ; \mu ; z ; p) t^{n}=(1-t)^{-\lambda}{ }_{2} F_{1, v}\left(\lambda, \eta ; \mu \frac{z}{1-t} ; p\right) .
$$

Proof. By considering the following series identity, we have

$$
[(1-z)-t]^{-\lambda}=(1-t)^{-\lambda}\left(1-\frac{z}{1-t}\right)^{-\lambda}
$$

Thus, the power series expansion yields

$$
\sum_{n=0}^{\infty} \frac{(\lambda)_{n}}{n !}(1-z)^{-\lambda}\left(\frac{t}{1-z}\right)^{n}=(1-t)^{-\lambda}\left[1-\frac{z}{1-t}\right]^{-\lambda}
$$

Multiplying both sides of (5.2) by $z^{\eta-1}$ and then applying the operator $\mathfrak{D}_{z ; v}^{\eta-\mu ; p}$ on both sides, we have

$$
\mathfrak{D}_{z ; v}^{\eta-\mu ; p}\left[\sum_{n=0}^{\infty} \frac{(\lambda)_{n}}{n !}(1-z)^{-\lambda}\left(\frac{t}{1-z}\right)^{n} z^{\eta-1}\right]=(1-t)^{-\lambda} \mathfrak{D}_{z ; v}^{\eta-\mu ; p}\left[z^{\eta-1}\left(1-\frac{z}{1-t}\right)^{-\lambda}\right] .
$$

Interchanging the order of summation and the operator $\mathfrak{D}_{z ; v}^{\eta-\mu ; p}$, we have

$$
\sum_{n=0}^{\infty} \frac{(\lambda)_{n}}{n !} \mathfrak{D}_{z ; v}^{\eta-\mu ; p}\left[z^{\eta-1}(1-z)^{-\lambda-n}\right] t^{n}=(1-t)^{-\lambda} \mathfrak{D}_{z ; v}^{\eta-\mu ; p}\left[z^{\eta-1}\left(1-\frac{z}{1-t}\right)^{-\lambda}\right] .
$$

Thus by applying Theorem 3.4, we obtain the required result. 
Theorem 5.2. The following generating relation holds true:

$$
\sum_{n=0}^{\infty} \frac{(\lambda)_{n}}{n !}{ }_{2} F_{1 ; v}(\delta-n, \eta ; \mu ; z ; p) t^{n}=(1-t)^{-\beta} F_{1}\left(\delta, \lambda, \eta ; \mu ;-\frac{z t}{1-t} ; p\right),
$$

where $|t|<\frac{1}{1+|t|}, m<\Re(\eta-\mu)<m<\Re(\eta)$.

Proof. Consider the series identity

$$
[1-(1-z) t]^{-\lambda}=(1-t)^{-\beta}\left[1+\frac{z t}{1-t}\right]^{-\lambda}
$$

Using the power series expansion to the left sides, we have

$$
\sum_{n=0}^{\infty} \frac{(\lambda)_{n}}{n !}(1-z)^{n} t^{n}=(1-t)^{-\lambda}\left[1-\frac{-z t}{1-t}\right]^{-\lambda}
$$

Multiplying both sides of (5.4) by $z^{\eta-1}(1-z)^{-\delta}$ and applying the operator $\mathfrak{D}_{z ; v}^{\eta-\mu ; p}$ on both sides, we have

$$
\mathfrak{D}_{z ; v}^{\eta-\mu ; p}\left[\sum_{n=0}^{\infty} \frac{(\lambda)_{n}}{n !} z^{\eta-1}(1-z)^{-\delta+n} t^{n}\right]=(1-t)^{-\lambda} \mathfrak{D}_{z ; v}^{\eta-\mu ; p}\left[z^{\eta-1}(1-z)^{-\delta}\left(1-\frac{-z t}{1-t}\right)^{-\lambda}\right],
$$

where $\Re(\eta)>\Re(\mu)>0$ and $|z t|<|1-t|$, thus by Theorem 3.2, we have

$$
\sum_{n=0}^{\infty} \frac{(\lambda)_{n}}{n !} \mathfrak{D}_{z ; v}^{\eta-\mu ; p}\left[z^{\eta-1}(1-z)^{-\delta+n}\right] t^{n}=(1-t)^{-\lambda} \mathfrak{D}_{z ; v}^{\eta-\mu ; p}\left[z^{\eta-1}(1-z)^{-\delta}\left(1-\frac{-z t}{1-t}\right)^{-\lambda}\right] .
$$

Applying Theorem 3.5 on both sides, we get the desired result.

\section{Concluding Remarks}

Recently, Kiymaz et. al. [6] introduced extended Caputo fractional derivative operator and they obtained many interesting results related to some familiar special functions by applying the said operator. In this paper, we established further extension of Kiymaz et. al. [6] Caputo fractional derivative operator and obtained many results related to some known special functions which are extensions of the work of Kiymaz et. al. We conclude that when $v=0$ then all the results established in this paper will reduce to the results associated with extended Caputo fractional derivative operator by using the term $K_{\frac{1}{2}}(z)=\sqrt{\frac{\pi}{2 z}} e^{-z}$

\section{REFERENCES}

[1] D. Baleanu, P. Agarwal, R. K. Parmar, M. M. Alquarashi, S. Salahshour, Extension of the fractional derivative operator of the Riemann-Liouville, J. Nonlinear Sci. Appl., 10(2017), 2914-2924.

[2] M. A. Chaudhry, A. Qadir, M. Rafique, S. M. Zubair, Extension of Eulers beta function, J. Comput. Appl. Math. 78 (1997) 1932.

[3] M. A. Chaudhry, A. Qadir, H. M. Srivastava and R. B. Paris, Extended hypergeometric and confluent hypergeometric functions, Appl. Math. Comput., 159 (2004) 589-602 
[4] J. Choi, A. K. Rathie, R. K. Parmar, Extension of extended beta, hypergeometric and confluent hypergeometric functions, Honam Mathematical J. 36 (2014), No. 2, pp. 357-385.

[5] S. A. Dar, R. B. Paris, A ( $p$,$) -extension of the Appell function F_{1}($.$) and its properties,$ arXiv:1711.07780 [math.CA], 2017.

[6] I. O. Kiymaz, A. Cetinkaya, P. Agarwal, An extension of Caputo fractional derivative operator and its application, J. Nonlinear Sci. Appl., 9 (2016), 3611-3621.

[7] A. A. Kilbas, H. M. Srivastava, J. J. Trujillo, Theory and application of fractional differential equation, North-Holland Mathematics Studies, Elsevier Sciences B.V., Amsterdam, (2006).

[8] M. J. Luo, G. V. Milovanovic, P. Agarwal, Some results on the extended beta and extended hypergeometric functions, Appl. Math. Comput., 248 (2014), 631-651.

[9] S. Mubeen, G. Rahman, K. S. Nisar, J. Choi. M. Arshad, An extended beta function and its properties, Far East Journal of Mathematical Sciences, 102(2017), 1545-1557.

[10] M. A. Özerslan, E. Özergin, Some generating relations for extended hypergeometric functions via generalized fractional derivative operator, Mathematical and Computer Modelling, 52 (2010), 1825-1833.

[11] M. A. Özerslan, E. Özergin, Extension of gamma, beta and hypergeometric functions, J. Comput. Appl. Math. 235 (2011), 4601-4610.

[12] F. W. J. Olver, D. W. Lozier, R. F. Boisvert, C. W. Clark (eds.), NIST handbook of mathematical functions, With 1 CD-ROM (Windows, Macintosh and UNIX), U.S. Department of Commerce, National Institute of Standards and Technology, Washington, DC; Cambridge University Press, Cambridge, (2010).

[13] T.R. Prabhakar, A singular integral equation with a generalized MittagLeffler function in the kernel, Yokohama Math. J. 19, 715, (1971).

[14] R. K. Parmar, Some generating relations for generalized extended hypergeometric functions involving generalized fractional derivative operator, J. Concr. Appl. Math., 12 (2014), 217-228.

[15] R. K. Parmar, P. Chopra, R. B. Paris, On an extension of extended beta and hypergeometric functions, arXiv:1502.06200 [math.CA], 22, 2015. [to appear in J. Classical Anal.]

[16] G. Rahman, S. Mubeen, K. S. Nisar, J. Choi, Extended special functions and fractional integral operator via an extended Beta function, Submitted.

[17] G. Rahman et al., Further extension of extended fractional derivative operator of the Riemann-Liouville, doi: 10.20944/preprints201712.0013.v1.

[18] E. D. Rainville, Special functions, The Macmillan Company, New York, 1960.

[19] S. G. Samko, A. A. Kilbas, O. I. Marichev, Fractional integrals and derivatives, Theory and applications, Edited and with a foreword by S. M. Nikoĺski , Translated from the 1987 Russian original, Revised by the authors, Gordon and Breach Science Publishers, Yverdon, (1993). 
[20] H. M. Srivastava, R. K. Parmar, P. Chopra, A class of extended fractional derivative operators and associated generating relations involving hypergeometric functions, Axioms, 1 (2012), 238-258.

Gauhar Rahman: Department of Mathematics, International Islamic University, IsLAMABAD, PAKISTAN

E-mail address: gauhar55uom@gmail.com

Kottakkaran Sooppy Nisar: Department of Mathematics, College of Arts and ScienceWadi Aldawaser, 11991, Prince Sattam bin Abdulaziz University,Alkharj, Kingdom of SAudi Arabia

E-mail address: n.sooppy@psau.edu.sa; ksnisar1@gmail.com

Muhammad Arshad

Department of Mathematics, International Islamic University, Islamabad, Pakistan

E-mail address: marshad_zia@yahoo.com 